\title{
La validez externa de las encuestas en la web. Amenazas y su control
}

\author{
Giorgio P. DE MARCHIS \\ Universidad Complutense de Madrid, España \\ giorgiodemarchis@ccinf.ucm.es
}

Recibido: $24 / 07 / 2012$

Aceptado: 17/10/2012

\begin{abstract}
Resumen
Es cada vez más frecuente que se lleven a cabo encuestas para la investigación y para el análisis de la docencia a través de la Web. Sin embargo, no conocemos artículos que delimiten la validez externa de los datos así obtenidos. Ésta se refiere a la capacidad de la investigación para generar resultados que se pueden generalizar. Proponemos analizar la literatura sobre la validez externa de encuestas hechas a través de internet, estableciendo cuáles son las amenazas potenciales, y sugerimos cómo controlarlas.

Palabras clave: validez, encuestas, internet, $W e b$, cuestionarios
\end{abstract}

\section{The external validity of surveys conducted via the web. Threats and control}

\begin{abstract}
It is increasingly common to conduct surveys for research and teaching analysis via the Web. However, we do not know of articles that delimit the external validity of the data thus obtained. External validity refers to the ability of research to produce outputs which can be generalized. We propose to analyze the literature on the external validity of surveys over the Internet, to establish what the potential threats are, and suggest how to control them.
\end{abstract}

Keywords: validity, surveys, internet, Web, questionnaires

\section{Referencia normalizada}

DE MARCHIS, Giorgio P. (2012): "La validez externa de las encuestas en la web. Amenazas y su control". Estudios sobre el mensaje periodístico. Vol. 18, núm. especial octubre, págs.: 263-272. Madrid, Servicio de Publicaciones de la Universidad Complutense.

Sumario: 1. Introducción. 2. Metodología. 3. Desarrollo; 3.1. Representatividad de la muestra; 3.2. Selección y Autoselección; 3.3. Mortalidad experimental; 3.4. Presentación automatizada de los estímulos; 3.5. Variables técnicas. 4. Conclusiones. 5. Referencias bibliográficas.

\section{Introducción}

Las innovaciones tecnológicas suelen llevar asociados cambios sociales, culturales, artísticos y científicos. Internet ha provocado, en mayor o menor medida, cambios en todos estos ámbitos. Los investigadores en Ciencias Sociales usan cada vez con más frecuencia las encuestas como herramienta para la recopilación de datos cuantitativos (Knoll et al., 2011). En la actualidad, muchas de las encuestas que se hacían en formato "papel y lápiz" se realizan a través de la Web. Internet ha aportado eficiencia en cuanto a ahorro de dinero y tiempo (Birnbaum, 2000), favoreciendo una democratización de la investigación (Kaplowitz et al., 2004) puesto que ya no se necesitan presupuestos elevados para realizar encuestas a gran escala (Couper y Miller, 2008; Loosveldt y Sonck, 2008). Además, con el mismo gasto se pueden conseguir datos de diferentes lugares del mundo y culturas. El ahorro de tiempo proviene de obtener los datos mucho más rápidamente que con el método presencial (Loosveldt y Sonck, 2008). Otra ventaja de las encuestas por internet es que los participantes pueden llevar a cabo el experimento 
cuando lo consideren oportuno, y en un entorno cómodo. Finalmente, internet permite hacer visibles algunos fenómenos psicológicos que no existen en el entorno natural o que allí son difíciles de estudiar. Por ejemplo el efecto de la desinhibición.

Sin embargo, todas estas ventajas serían inútiles si los estudios hechos por internet no tuvieran validez. Pues, toda investigación científica requiere tenerla.

Se han escrito artículos de revisión sobre las encuestas por internet (Couper, 2000; Frippiat y Marquis, 2010), pero ninguno se ha centrado exclusivamente en los aspectos de la validez. En estas páginas nos proponemos llevar a cabo un estudio de la literatura existente sobre la realización de encuestas a través de internet, $\mathrm{y}$, analizarla desde la perspectiva de su validez externa. También se ofrecen sugerencias sobre cómo aumentar la validez externa de las encuestas a través de la Web. Esta tarea es importante porque puede ayudar a mejorar el diseño de nuevos estudios, y auxiliar al análisis crítico de los resultados y conclusiones de investigaciones que usan esta herramienta metodológica.

\section{Metodología}

La búsqueda de la bibliografía relevante se ha llevado a cabo en la base de datos Communication and Mass Media Complete. Se han seleccionado los documentos publicados entre el año 2000 y Julio 2012 mediante la búsqueda de los términos "valid", junto con "survey", "web", o "internet". Se han seleccionado aproximadamente sesenta artículos, que son los que finalmente se han revisado. La exploración completa de estos artículos ha llevado a la revisión de otros que en un primer momento no estaban seleccionados.

\section{Desarrollo}

El concepto de validez se define como la capacidad de la investigación para "mostrarnos o representar esa realidad a la que se refiere" (Sierra Bravo; 2001: 126), esto es, el grado de veracidad de los resultados obtenidos. A lo largo de varias publicaciones seminales, Campbell y colegas (Campbell y Stanley, 1963; T. D. Cook y Campbell, 1979; Shadish et al., 2002) definieron el concepto, y clasificaron varios tipos de validez. La validez externa, que es la que aquí nos interesa, se refiere a la capacidad de generalizar los resultados, y por ello es especialmente importante en los estudios que quieren describir una realidad.

\subsection{Representatividad de la muestra}

Generalizar es la posibilidad de extrapolar las conclusiones de una muestra hacia poblaciones más grandes (Brenner, 2002), y sólo se puede hacer si la muestra es representativa. Si no lo es, los participantes no contestan como el resto de miembros de la población responderían, y existe un sesgo de validez poblacional.

Internet permite obtener muestras de gran tamaño, pero esto no significa que sean representativas. Por ejemplo, no todo el mundo tiene acceso a internet (Best y Krueger, 2004), y puede haber importantes diferencias entre el acceso a internet de la población de las ciudades y del campo; de las personas con capacidad adquisitiva media y baja; de los inmigrantes; o entre países desarrollados y en vida de desarrollo. En el año 
1936, la revista Reader's Digest hizo una encuesta con una muestra de 2 millones de lectores basada en una población de 10 millones de personas que poseían teléfono y coche. La encuesta predijo la victoria de Alf Landon en las presidenciales. Franklin Roosevelt fue presidente. El error, posiblemente, fuera debido a que la mayoría de las personas que tenían teléfono y coche eran votantes de Landon. Así, la muestra no representaba la realidad de EE.UU. en aquella época (Larose y Atkin, 1992). Igualmente, en los comienzos de internet había un sesgo de representación, al ser los primeros usuarios de internet varones con educación y de clase medio alta (Don A. Dillman, 2000). Hoy en día, por ejemplo, los participantes en paneles es más probable que sean varones jóvenes con un elevado nivel educativo (Lensvelt-Mulders y Hubregtse, 2009). Por ello hay que tener cuidado a la hora comparar los resultados de estudios realizados con muestras procedentes de diferentes fuentes.

De todas formas, no olvidemos que la mayoría de las investigaciones académicas, tanto offline como online, usan muestras de conveniencia (Skitka y Sargis, 2006). Y en las muestras universitarias suele haber una mayor proporción de mujeres de edades comprendidas entre los 18 y los 22 años (Birnbaum, 2000). Sin embargo, este hecho no la impedido el avance de una ciencia 'válida' (Mook, 1983), entre otras cosas porque con el aumento de la muestra aumenta la potencia estadística. Así, el secreto de la representatividad parece estar en la repetición.

\subsection{Selección y Autoselección}

Si el diseño de la investigación requiere de la comparación entre diferentes grupos o estudios, es necesario controlar la equivalencia inicial de las unidades que se comparan. Para que se tenga esta equivalencia inicial se usa la aleatorización de los participantes. Una vez hecha la selección se pueden asignar diferentes cuestionarios de forma aleatoria. En la literatura se señala cómo mejorar la selección de la muestra en las investigaciones online (Medders, 2010). Por ejemplo, se puede seleccionar una población cuyos miembros estén en listados accesibles de internet (Couper, 2000), como los miembros de páginas web que favorecen encontrar pareja. Se pueden seleccionar participantes de listas de interés (Cho et al., 2001), o se puede usar Facebook. Pero nada puede asegurar que la identidad de los participantes sea la auténtica (Englis y Solomon, 2000), o que una persona no tenga varias identidades o que no esté en varias listas y que por ello sus datos se dupliquen (Fisher et al., 1996).

$\mathrm{Si}$, en cambio, los participantes leen la invitación a través de anuncios en grupos de discusión, comunidades online, banners, etc., y deciden participar o no, estamos usando un método autoselectivo. La autoselección provoca sesgos que son difíciles de superar (Loosveldt y Sonck, 2008), además de que a menudo pasan desapercibidos. Las personas que eligen participar en la encuesta pueden tener un perfil determinado. Varias investigaciones han demostrado (Chang y Krosnick, 2009; Shropshire et al., 2009) que las personas que llevan a cabo una encuesta pagada o autoseleccionada por internet tienen más conocimientos, compromiso o afinidad con el tema de la encuesta tratado que las personas que habían sido elegidas de forma aleatoria. Parece lógico pensar que una persona que tiene más interés y compromiso con el tema de la encuesta tiene más probabilidades de iniciarla, y terminarla. 
Este sesgo se puede superar si se tiene información sobre cómo debería ser la población a investigar. Reips (2002b) sugiere poner unas etiquetas invisibles y diferentes en cada cuestionario colgado en las páginas web o enviado por email, de manera que se podría conocer la proveniencia de cada participante. Así, se podría ir seleccionando y descartando los datos de determinados perfiles hasta obtener una muestra representativa. Las discrepancias entre grupos se pueden usar para medir y corregir los sesgos. Si no hay datos sobre la población, es difícil evitar este sesgo (Frippiat y Marquis, 2010).

\subsection{Mortalidad experimental}

La expresión 'mortalidad experimental' se refiere a la pérdida de participantes a lo largo del proceso de investigación. Se trata de un problema de validez muy frecuente en las encuestas a través de internet. Este sesgo lleva a una falta de proporcionalidad entre los diferentes grupos de participantes. La tasa de respuesta y el abandono son conceptos relacionados con la mortalidad experimental.

La tasa de respuesta es el ratio entre el número de respuestas y el número total de solicitudes de participación enviadas. En los estudios por internet, la tasa de respuesta también se puede calcular como el ratio entre los que no han contestado del total que han llegado a la página de la encuesta. Baxter y Babbie (2004) sugieren como regla de oro que una tasa de respuesta del $50 \%$ es adecuada, un $60 \%$ es buena, y un $70 \%$ es muy buena. Tener una tasa baja puede llevar a una pérdida la representatividad de la muestra. Se ha calculado que cuando se solicita una participación por correo electrónico, el porcentaje de respuesta ronda el 10\% (Couper, 2000), que es un porcentaje muy bajo para poder generalizar los resultados. Para mejorar la tasa de respuesta, se ha sugerido notificar que se va a llevar a cabo el experimento y hacer un seguimiento del contacto para su participación (Palomares y Flanagin, 2005). Algunas investigaciones han encontrado que ambas acciones influyen en la tasa de respuesta (C. Cook et al., 2000), aunque otras (Muñoz-Leiva et al., 2010) han encontrado que la frecuencia de los contactos no ha llevado a efectos significativos. La explicación de los autores es que los receptores se protegen activando el filtro antispam.

Algunos autores (Smyth et al., 2010) han sugerido que para compensar las bajas tasas de respuesta de los cuestionarios por correo electrónico se pueden mandar cuestionarios postales unas semanas después del contacto por email. Rookey y col. (2008) han encontrado que el perfil socio-demográfico de las personas que contestan a las encuestas por internet es diferente del que tienen las personas que contestan a los cuestionarios por correo, aumentando así la representatividad de la muestra. Dillman y col. (2009) han encontrado eficaz realizar una encuesta telefónica entre las personas que no habían contestado a una encuesta por internet, al pasar de una tasa de respuesta del $12,7 \%$ en internet a una del $44,9 \%$ por teléfono. En este estudio no hubo un impacto significativo en la distribución de las variables socio-demográficas. El procedimiento descrito es útil siempre que no haya sesgos provocados por los instrumentos, porque los datos se deben unir como si fueran parte de la misma muestra.

En cambio parece ser que es poco eficaz mandar primero el correo postal, y tres semanas después ofrecer la posibilidad de respuesta por internet (Don A. Dillman et 
al., 2010), o dejar elegir al participante entre el modo de respuesta online o por correo (De Leeuw, 2005).

Otros consejos para aumentar la tasa de respuesta es dirigirse a los participantes por su nombre (Joinson y Reips, 2007); prometer compartir los resultados o dar retroalimentación, y avisar de que se trata de un estudio académico, no comercial (Bosjnak y Batinic, 2002).

El abandono es otro problema relacionado. Es infrecuente que alguien que lleve a cabo una investigación presencial se vaya antes de terminarla, en cambio es muy común que en internet los sujetos no terminen la encuesta. Uno de los problemas del abandono es que la muestra puede quedar sesgada, puesto que las personas que abandonan pueden hacerlo por tener poco interés en el tema de la encuesta, o por tener una determinada actitud. Este problema es especialmente acuciante en la recogida de datos longitudinales, pues es más difícil mantener el contacto con los participantes a través de internet que por teléfono.

Se han diseñado estrategias para reducir el abandono. Puesto que son varias, las listaré: (a) Dar incentivos económicos. (b) Solicitar información personal, como sexo, edad, nacionalidad, dirección, número de teléfono antes de comenzar el experimento. porque al final no suelen darlos (Frick et al., 2001). (c) Pedir al encuestado dar la opinión que tenga de la institución que lleva a cabo el experimento. (d) Preguntar a los participantes con qué probabilidad van a terminar de contestar al estudio. (e) Poner imágenes de la persona solicitante de la encuesta (Guégen y Jacob, 2002). (f) Hacer un diseño de las páginas web atractivo y cómodo. (g) Tener un sistema informático rápido, sin tiempos de espera mayores de tres segundos (Meyer et al., 1999). (h) Uso de un lenguaje simple. En una investigación llevada a cabo por internet y con más de once mil participantes (Ganassali, 2008) se averiguó que la tasa de abandono pasaba de $29 \%$ a $23 \%$ si se simplificaba el lenguaje. (i) Reducción de la duración percibida del cuestionario, por ejemplo avisando al comienzo que el cuestionario va a ser de 20 o de 40 preguntas. Pues, el porcentaje de abandono baja del 34\% al 21\% (Ganassali, 2008). (j) Describir el estudio en detalle antes de comenzarlo. Se ha averiguado que es más probable que los participantes dejen el cuestionario sin terminar si tienen poco interés en el tema sobre el que se pregunta (Shropshire et al., 2009), y que, al contrario, es más probable que dediquen tiempo y energía si están interesados en el tema (Couper, 2000). Por supuesto, esta conducta lleva a un sesgo peligroso, esto es, que las personas que contesten son las que tienen alta implicación. Por supuesto, (k) es necesario usar un idioma comprensible por el entrevistado.

Todas estas técnicas están relacionadas con la motivación (Reips, 2002a), y por ello su uso amenaza el estudio de las variables motivacionales de los participantes.

\subsection{Presentación automatizada de los estímulos}

Presentar los estímulos a través de un ordenador, sea online u offline, permite mostrar los estímulos de forma progresiva. Esto tiene tres ventajas (Frippiat y Marquis, 2010). La primera es que se evita que los participantes miren las preguntas antes de contestar o vuelvan hacia atrás para cambiar las respuestas dadas. La segunda es que se pueden presentar las preguntas de manera tal que el ritmo de la presentación del 
cuestionario sea el deseado. Finalmente, se pueden presentar las preguntas en función de las respuestas. De esta manera los encuestados no se pierden, confunden o dejan de contestar a los apartados de 'si contestas...entonces ve a la pregunta...', puesto que este proceso lo controla el programa, sin que el encuestado se dé cuenta.

Tanto la tasa de respuesta como la tasa de abandono se pueden mejorar a través de los incentivos materiales (Göritz, 2006). Los incentivos funcionan mejor si están desligados de la realización de la encuesta, y son un beneficio sin contrapartida directa (Sánchez-Fernández et al., 2010). Por ejemplo, ofrecer una pequeña cantidad como 5 Euros con la solicitud aumenta significativamente la tasa de respuesta en las encuestas online (Messer y Dillman, 2010). Los incentivos condicionados a la respuesta del cuestionario no tienen un efecto relevante para que los participantes inicien a contestar, pero lo tienen para que terminen de contestar (Sánchez-Fernández et al., 2010). Los incentivos no influyen en el sesgo de representatividad (Sanchez-Fernandez et al., 2012).

\subsection{Variables técnicas}

Si se quieren medir variables dependientes (p.ej. los tiempos de respuesta), hay que tener en cuenta que no se pueden controlar muchas variables técnicas. Algunos ejemplos son la velocidad de acceso (p.ej. navegador, tipo de conexión) y la velocidad del equipamiento informático. Aunque las limitaciones tecnológicas en los países occidentales son cada vez menores, siguen siendo variables que hay que controlar a la hora de analizar la validez de los datos.

\section{Conclusiones}

Puede que las razones básicas para llevar a cabo investigaciones por internet sean el ahorro de tiempo y esfuerzo en conseguir datos, y el abaratar costes, más que aumentar la validez. Además, la metodología se tiene que ajustar a los usos y costumbres de las personas. Y hoy en día las personas usan internet para comunicar. Sea la razón que sea por la que los investigadores usan las encuestas por internet, todo investigador tiene que preocuparse por la validez de sus investigaciones.

En este artículo se han listado una serie de aspectos que conforman la validez de las investigaciones en internet, al tiempo que se han sugerido soluciones. Muchas de las sugerencias que se pueden hacer para aumentar la validez son similares a las que se aconseja llevar a cabo en las investigaciones tradicionales, de 'papel y lápiz', mientras que otras son idiosincráticas de este medio.

En general, se sugiere el uso de grupos de control, contrabalancear los tratamientos, y emplear de forma simultánea varias técnicas de obtención de información, para comparar los resultados.

Conocer la validez de un instrumento requiere tiempo. Los resultados encontrados hasta ahora sobre la validez de los estudios por internet son esperanzadores, sin embargo sólo tras llevar a cabo más investigaciones que ofrezcan similares resultados se podrá llegar a conclusiones fiables las diferentes facetas de la validez.

También es necesario seguir investigando para hallar nuevaxteras formas para aumentar la validez de los estudios hechos a través de la red de redes. 


\section{Referencias bibliográficas}

BAXTER, Leslie A., \& BABBIE, Earl R. (2004). The basics of communication research. Belmont, CA: Thomson Learning.

BEST, Samuel J., \& KRUEGER, Brian S. (2004). Internet Data Collection. Quantitative Applications in the Social Sciences. London: Sage publications.

BIRNBAUM, Michael H. (2000). Psychological experiments on the Internet. San Diego: Academic Press.

BOSJNAK, Michael, \& BATINIC, Bernard (2002). Understanding the willingness to participate in online-surveys. The case of e-mail questionnaires. En Bernard BATINIC, Ulf-Dietrich REIPS \& Michael BOSNJAK (Eds.), Online social sciences (pp. 81-92). Seattle, WA: Hogrefe \& Huber.

BRENNER, Viktor. (2002). Generalizability issues in Internet-based survey research: implications for the Internet addiction controversy. En Bernard BATINIC, Ulf-Dietrich REIPS \& Michael BOSNJAK (Eds.), Online social sciences (pp. 93-114). Seattle, WA: Hogrefe \& Huber.

CAMPBELL, Donald, \& STANLEY, Julian. (1963). Experimental and quasi-experimental designs for research. Chicago, IL: Rand-McNally.

COOK, Colleen, HEATH, Fred, \& THOMPSON, Russel L. (2000). A meta-analysis of response rates in web- or internet-based surveys. Educational and Psychological Measurement, 60, 821-836.

COOK, Thomas D., \& CAMPBELL, Donald. (1979). Quasi-experimentation: Design and analysis issues for field settings. Boston, MA: Houghton Mifflin Company.

COUPER, Mick P. (2000). Web surveys - A review of issues and approaches. Public Opinion Quarterly, 64(4), 464-494. doi: 10.1086/318641

COUPER, Mick P., \& MILLER, Peter V. (2008). Web survey methods. Introduction. Public Opinion Quarterly, 72(5), 831-835. doi: 10.1093/poq/nfn066

CHANG, LinChiat, \& KROSNICK, Jon A. (2009). National Surveys via RDD Telephone versus the Internet: Comparing Sample Representativeness and Response Quality. Public Opinion Quarterly, 73(4), 641-678.

CHO, Chang-Hoan, LEE, Jung-Gyo, \& THARP, Marye C. (2001). The Effects of Different Forced Exposure Levels to Banner Advertisements on the WWW. Journal of Advertising Research, 41(July/August), 45-56.

DE LEEUW, E. D. (2005). To mix or not to mix data collection modes in surveys. Journal of Official Statistics, 21, 233-255.

DILLMAN, Don A. . (2000). Mail and Internet Surveys: The Tailored Design Method. New York: John Wiley \& Sons.

DILLMAN, Don A., PHELPS, Glenn, TORTORA, Robert, SWIFT, Karen, KOHRELL, Julie, BERCK, Jodi, et al. (2009). Response rate and measurement differences in mixed-mode surveys using mail, telephone, interactive voice response (IVR) and the Internet. Social Science Research, 38(1), 1-18. 
DILLMAN, Don A., REIPS, Ulf-Dietrich, \& MATZAT, Uwe. (2010). Editorial. International Journal of Internet Science, 5(1), 1-4.

ENGLIS, Basil G., \& SOLOMON, Michael R. (2000). Life/Style Online C. A webbased methodology for visually-oriented consumer research. Journal of Interactive Marketing, 14(1), 2-14.

FISHER, Bonnie, MARGOLIS, Michael, \& RESNIK, David. (1996). Surveying the Internet: Democratic Theory and Civic Life in Cyberspace. Southeastern Political Review, 24(3).

FRICK, Andrea, BÄCHTIGER, Marie-Therese, \& REIPS, Ulf-Dietrich. (2001). Financial incentives, personal information and drop-out in online studies. En UlfDietrich REIPS \& Michael BOSNJAK (Eds.), Dimensions of Internet Science (pp. 209-219). Lengerich: Pabst.

FRIPPIAT, Didier, \& MARQUIS, Nicolas. (2010). Web Surveys in the Social Sciences: An Overview. Population, 65(2).

GANASSALI, Stephane. (2008). The influence of the design of web survey questionnaires on the quality of responses. Survey Research Methods, 2(1), 21-32.

GÖRITZ, Anja S. (2006). Incentives in web studies: Methodological issues and a review. International Journal of Internet Science, 1(1), 58-70.

GUÉGEN, Nicolas, \& JACOB, Céline. (2002). Social presence reinforcement and computer-mediated communication: the effect of the solicitor's photography on compliance to a survey request made by e-mail. Cyber-Psychology and Behavior, $5,139-142$.

JOINSON, Adam N., \& REIPS, Ulf-Dietrich. (2007). Personalized salutation, power of sender and response rates to web-based surveys. Computers in Human Behavior, 23(3), 1372-1383.

KAPLOWITZ, Michael D., HADLOCK, Timothy D., \& LEVINE, Ralph. (2004). A comparison of web and mail survey response rates. Public Opinion Quarterly, 68(1), 94-101.

KNOLL, Monja A., UTHER, Maria, \& COSTALL, Alan. (2011). Using the Internet for speech research: an evaluative study examining affect in speech. Behaviour \& Information Technology, 30(6), 845-851.

LAROSE, Robert, \& ATKIN, David. (1992). Audiotext and the reinvention of the telephone as a mass medium. Journalism Quarterly (69), 413-421.

LENSVELT-MULDERS, Gerty J. L. M., LUGTIG, Peter J., \& HUBREGTSE, Marianne. (2009). Separating Selection Bias and Non-coverage in Internet Panels using Propensity Matching. Survey Practice (August).

LOOSVELDT, Geert, \& SONCK, Nathalie. (2008). An evaluation of the weighting procedures for an online access panel survey. Survey Research Methods, 2(2), 93105. 
MEDDERS, Ryan. (2010). Conducting Social Scientific Research on the Internet: A Comparison of Offline and Online Methods. Paper presented at the Paper presented at the annual meeting of the International Communication Association, Suntec Singapore International Convention \& Exhibition Centre, Suntec City, Singapore. http://www.allacademic.com/meta/p404680_index.html

MESSER, Benjamin L., \& DILLMAN, Don A. (2010). Using address based sampling to survey the general public by mail vs. "Web plus mail" (pp. 1-55). Pullman, WA: Social and Economic Sciences Research Center, Washington State University.

MEYER, Herbert A., HÄNZE, Martin, GÜNTEKIN, Ebru, GREBE, Martin, BREDE, Pia, \& HILDEBRANDT, Michael. (1999). Computers as pacemakers: The influence of system response time on navigating trough hypertext. En Ulf-Dietrich REIPS, Bernad BATINIC, Wolfgang BANDILLA, Michael BOSNJAK, Lorenz GRÄF, Klaus MOSER \& Andreas WERNER (Eds.), Current Internet science trends, techniques, results. Zürich: Online Press.

MOOK, Douglas G. (1983). In defense of external invalidity. American Psychologist, 38, 379-387.

MUÑOZ-LEIVA, Francisco, SÁNCHEZ-FERNÁNDEZ, Juan, MONTORO-RÍOS, Francisco, \& IBÁÑEZ-ZAPATA, José Ángel. (2010). Improving the response rate and quality in web-based surveys through the personalizationand frequency of reminder mailings. Quality and Quantity, 44(5), 1037-1052.

PALOMARES, Nicholas A., \& FLANAGIN, Andrew J. (2005). The potential of electronic communication and information technologies as research tools: Promise and perils for the future of communication research. En Pamela J. KALBFLEISCH (Ed.), Communication Yearbook (Vol. 29, pp. 147-185). Mahwah, NJ: Lawrence Erlbaum.

REIPS, Ulf-Dietrich. (2002a). Standards for internet-based experimenting. Experimental Psychology, 49, 243-256.

REIPS, Ulf-Dietrich. (2002b). Theory and techniques of Web experimenting. En Bernad BATINIC, Ulf-Dietrich REIPS \& Michael BOSNJAK (Eds.), Online social sciences (pp. 229-250). Seattle: Hogrefe \& Huber.

ROOKEY, Bryan D., HANWAY, Steve, \& DILLMAN, Don A. (2008). Does a probability-based household panel benefit from assignment to postal response as an alternative to internet-only? Public Opinion Quarterly, 72(5), 962-984.

SÁNCHEZ-FERNÁNDEZ, Juan, MUNOZ-LEIVA, Francisco, \& MONTORO-RÍOS, Francisco Javier. (2012). Improving retention rate and response quality in Webbased surveys. Computers in Human Behavior, 28(2), 507-514. doi: 10.1016/j.chb.2011.10.023

SÁNCHEZ-FERNÁNDEZ, Juan, MUÑOZ-LEIVA, Francisco, MONTORO-RÍOS, Francisco Javier, \& IBÁÑEZ-ZAPATA, José Ángel. (2010). An analysis of the effect of pre-incentives and post-incentives based on draws on response to web surveys. Quality and Quantity, 44(2), 357-373. 
SHADISH, William R., COOK, Thomas D., \& CAMPBELL, Donald. (2002). Experimental and quasi-experimental designs for generalized causal inference. Boston, MA: Houghton Mifflin.

SHROPSHIRE, Kevin O. , HAWDON, James E. , \& WITTE, James C. (2009). Web survey design: Balancing measurement, response, and topical interest. Sociological Methods and Research, 37(3), 344-370.

SKITKA, Linda J., \& SARGIS, Edward G. (2006). The Internet as a Psychological Laboratory. Annual Review of Psychology, 57(1), 529-555.

SMYTH, Jolene D., DILLMAN, Don A., CHRISTIAN, Leah Melani, \& O'NEILL, Allison C. (2010). Using the Internet to survey small towns and communities: Limitations and possibilities in the early 21 st century. American Behavioral Scientist, $53,1423-1448$.

\section{Giorgio P. DE MARCHIS}

giorgiodemarchis@ccinf.ucm.es

Universidad Complutense de Madrid

Avda. de la Complutense s/n

28040, Madrid

Profesor de Psicología de la Comunicación y Creatividad 